\title{
The effect of current crowding on the heat and light pattern in high-power AIGaAs light emitting diodes
}

\author{
A. V. Zinovchuk, ${ }^{1}$ O. Yu. Malyutenko, ${ }^{1, a)}$ V. K. Malyutenko, ${ }^{1}$ A. D. Podoltsev, ${ }^{2}$ and \\ A. A. Vilisov ${ }^{3}$ \\ ${ }^{1}$ Lashkaryov Institute of Semiconductor Physics, NAS of Ukraine, 41 Prospekt Nauky, Kiev 03028, Ukraine \\ ${ }^{2}$ Institute of Electrodynamics, NAS of Ukraine, 56 Peremogy Avenue, Kiev 03680, Ukraine \\ ${ }^{3}$ Institute of Semiconductor Devices, 99-A Krasnoarmeiskaya Street, Tomsk 634034, Russia
}

(Received 11 January 2008; accepted 12 June 2008; published online 13 August 2008)

\begin{abstract}
The results of the light and temperature micromapping in AlGaAs light emitting diodes grown by liquid phase epitaxy as double heterostructures and emitting at $\lambda \sim 0.87 \mu \mathrm{m}$ are presented. At a driving current well above the safe operating limit $(>300 \mathrm{~mA})$, the nonuniform light pattern and local self-heating (with temperature gradient of about $950{ }^{\circ} \mathrm{C} / \mathrm{cm}$ ) followed by catastrophic degradation of a device were detected with the charge coupled device and infrared microscopes operating in a pulsed mode. These were shown to result from the current crowding effect in the active and contact areas of a device. Good agreement between the theory and experiment was found. (C) 2008 American Institute of Physics. [DOI: 10.1063/1.2968220]
\end{abstract}

\section{INTRODUCTION}

The AlGaAs/GaAs high-power light emitting diodes (LEDs) and lasers that emit in the near-infrared (IR) band have great potential for a variety of cost-effective practical applications such as industrial IR equipment, consumer electronics, high-speed fiber optic communications, and optical pumping of solid state lasers. For this reason, the properties of AlGaAs/GaAs have been studied thoroughly and it remains the best developed material for near-IR optoelectronics. By now, commercial LEDs have been optimized to such an extent that at $T=300 \mathrm{~K}$ and low injection levels (bias current $I<100 \mathrm{~mA})$, the internal quantum efficiency is $>98 \%$ (Refs. 1 and 2), and Joule heating (JH) can be neglected even in continuous wave (cw) mode.

However, at higher injection levels, some problems cause the efficiency of AlGaAs/GaAs emitting devices to decline. In most cases, shortcomings are related to the removal of heat, ${ }^{3}$ which is due to the $\mathrm{JH}$ of the structure and increased rate of nonradiative Auger recombination of injected charge carriers. In our opinion, the appearance of the current crowding (CC) effect, ${ }^{4}$ which is rarely mentioned in $\mathrm{AlGaAs} / \mathrm{GaAs}$ structures $^{5}$ but is well established in less efficient blueshifted $^{6-8}$ and redshifted ${ }^{9-12}$ LEDs, should also be considered. This effect is due to the localization of the current flow rout in some regions of a multilayer LED structure whose position and geometry are difficult to predict $a$ priori. As a result, the activation of the nonradiative recombination rate and the overheating of the structure can occur in these regions, along with a decrease in LED internal quantum efficiency (dynamic reversible degradation). Because of a low thermal conductivity of III-V compounds, the heat "traps" and dangerous temperature gradients can also form. These factors seem to prevent a high-output $\mathrm{cw}$ operation and to cause a catastrophic irreversible degradation of the LEDs as well. It is very important to note that increasing the diameter of the active area does not boost the output power because of

\footnotetext{
a)Electronic mail: malyut@isp.kiev.ua.
}

the $\mathrm{CC}$ effect. Furthermore, it has been recently shown that in the 3-5 $\mu \mathrm{m}$ LEDs that are made of InAsSb heterostructures (the internal quantum efficiency is $20 \%$ ), the CC forms dangerously high $\left(\sim 3000{ }^{\circ} \mathrm{C} / \mathrm{cm}\right)$ temperature gradients and reduces the emitting area by several times. ${ }^{12,13}$ The catastrophic degradation of blue InGaN quantum well LEDs also occurs in the CC region. ${ }^{14}$

General trends in commercial AlGaAs-based LEDs have been toward a higher output, a high quality light beam, and a longer operation time. Therefore, the attempts to minimize the CC effect seem to be of the most urgency. Meanwhile, the impact of the CC on AlGaAs LED performance has not been reported in details earlier. In this paper, we study this effect in LEDs made of double heterostructures, which are optimal for the design and efficiency due to both photon and charge carrier confinements. With this goal in mind, we investigated the two-dimensional (2D) distribution of local self-heating and the electroluminescence pattern in microscale using a separate detection of radiation that escapes the device in two spectral bands. The thermal radiation (that is due to the self-heating) was detected in the mid-IR ( $\lambda$ $=3-5 \mu \mathrm{m}$ ) band, while the recombination light (electroluminescence) output was detected in the fundamental absorption band $(\lambda \leq 1 \mu \mathrm{m})$. To ensure a spatial localization of heat sources, the measurements were performed in a pulsed mode. As a result, we have "visualized" those areas of the LED structure where CC induces local heating and nonuniformity in the electroluminescence output. By working in the overdrive mode, we have determined the temperatures of local overheating and temperature gradients inside the structure. We have also demonstrated that catastrophic degradation only happens in the region where the current crowds. The experimentally obtained results were compared to simulations carried out by the finite element method.

\section{LED DESIGN AND PARAMETERS}

Our study is concerned with the testing of AlGaAs LEDs grown by liquid-phase epitaxy as double heterostructures on 


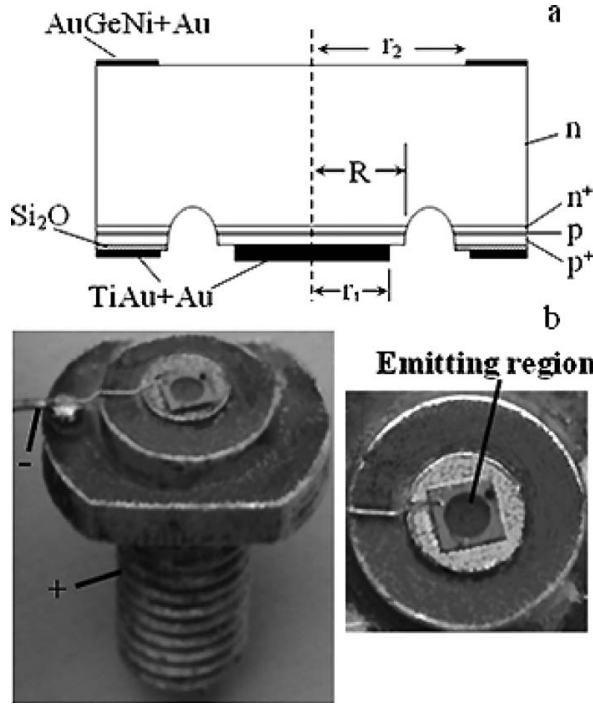

FIG. 1. (a) Schematic cross section of a multilayer AlGaAs emitting structure. (b) General view of the LED chip sited on a massive heat sink.

the $n$-GaAs substrate that was separated from the emitting structure at the last stage of $1.2 \times 1.2 \mathrm{~mm}^{2}$ chip fabrication. The emitting (at the wavelength $\lambda=0.87 \mu \mathrm{m}$ ) area was a 2 - $\mu$ m-thick $p$ - $\mathrm{Al}_{0.04} \mathrm{Ga}_{0.96} \mathrm{As}(\mathrm{Ge})$ active layer $\quad\left(p_{\mathrm{Ge}}=1\right.$ $\left.\times 10^{17} \mathrm{~cm}^{-3}\right)$, and it was located between a wide band gap 10 - $\mu$ m-thick $p^{+}-\mathrm{Al}_{0.25} \mathrm{Ga}_{0.75} \mathrm{As}\left(p_{\mathrm{Ge}}^{+}=1.5 \times 10^{18} \mathrm{~cm}^{-3}\right)$ and 5 - $\mu$ m-thick $n^{+}-\mathrm{Al}_{0.25} \mathrm{Ga}_{0.75} \mathrm{As}(\mathrm{Te}) \quad\left(n_{\mathrm{Te}}^{+}=3.9 \times 10^{17} \mathrm{~cm}^{-3}\right)$ confining layers, followed by a $140 \mu \mathrm{m} n-\mathrm{Al}_{0.25} \mathrm{Ga}_{0.75} \mathrm{As}$ $\left(n_{\mathrm{Te}}=1.7 \times 10^{17} \mathrm{~cm}^{-3}\right)$ buffer layer [Fig. 1(a)]. These structures were prepared as large-area circular mesas $(R$ $=250 \mu \mathrm{m})$. The contact areas $r_{1} \leq 220 \mu \mathrm{m}(\mathrm{TiAu}+\mathrm{Au})$ and $r_{2} \geq 525 \mu \mathrm{m}(\mathrm{AuGeNi}+\mathrm{Au})$ were located on opposite faces of the structure in a way that ensured a large area of the bottom central contact and prevented the top contact shadowing of light that was escaping the structure through the thick buffer $n$-layer. To ensure an efficient removal of the excess heat, the emitting chip was located in a diode package with a thin $p^{+}$-layer downward. Finally, the package itself was made as a massive copper heat sink holder [Fig. 1(b)].

From the analysis of the voltage-current dependence it was determined that the LED $p-n$-junction had the ideality factor of about 1.6 and the series resistance of $\sim 0.5 \Omega$. The thermal resistance of the structure (determined from the dependence of heating temperature on electric power) was $\sim 7{ }^{\circ} \mathrm{C} / \mathrm{W}$, and the heat relaxation time did not exceed $6 \mathrm{~ms}$. In $\mathrm{cw}$ mode without forced cooling, the light output was almost linearly proportional to the current up to a value of $I=350 \mathrm{~mA}$. At that current, the emission power exceeded 30 $\mathrm{mW}$. However, it decreased gradually when the temperature increased (thermal quenching). To illustrate this, as LED temperature grew by $50{ }^{\circ} \mathrm{C}$, the emission power decreased by $25 \%$, while it was almost halved at heating by $100{ }^{\circ} \mathrm{C}$ (these tests were made by mounting a device on a temperature-controlled heater and driving it at a current of 20 $\mathrm{mA})$.

\section{TWO-SPECTRAL MEASUREMENT PROCEDURE}

The heat pattern and local temperatures were characterized by the test system that consisted of an IR scanning cam- era equipped with an IR microscope that was operated in the 3-5 $\mu \mathrm{m}$ band (temperature resolution of $0.1^{\circ} \mathrm{C}$, spatial resolution of $<20 \mu \mathrm{m})$. To localize heat sources in the structure, these measurements were performed in the pulsed mode with a frame exposition time $\Delta t$ that ranged from $400 \mu$ s to $160 \mathrm{~ms}$. The calibrated IR camera was synchronized with the driving current $(I)$ in such a way that the first frame recorded the equilibrium distribution of the LED thermal radiation corresponding to its temperature $(I=0)$, while the second frame recorded the heat pattern at $I>0$. By subtracting the first frame from the second one, an image was obtained that showed the 2D heat distribution caused by the current. A special program transformed the power registered by each of the 19600 points (from which the image is formed) to a local temperature value. This program also "corrected" the nonuniformity of the emissivity over the surface using a socalled equalization procedure. The IR microscope was focused directly at the LED emitting area; therefore, the "thermal signal" was partially absorbed by free charge carriers in the $140-\mu \mathrm{m}$-thick buffer $n$-layer. According to our estimations, the inaccuracy in determining the temperature did not exceed $10 \%$, so it was not taken into account.

The electroluminescence pattern was recorded using a parallel video channel with a conventional charge coupled device (CCD) camera that was equipped with a microscope (operating wavelength band of $0.4-1.0 \mu \mathrm{m}$, spatial resolution of about $5 \mu \mathrm{m}$ ). To avoid the signal saturation at a high bias current, a set of calibrated gray filters was used. All tests were performed while pushing the devices beyond their safe operating limit.

\section{SIMULATION OF CURRENT AND HEAT SPREADING}

A numerical 2D modeling of the current flow through a multilayer structure and a 2D heat pattern in each layer was performed with a finite element simulation. The cylindrical model for calculations (with z-axis normal to the layer planes) involved the following four regions: (i) $p^{+}$-layer, (ii) $p$ - $n$-junction, (iii) active $p$-layer, and (iv) $n$-layer $\left(n^{+}\right.$-layer and buffer $n$-layer are presented as a single $145-\mu \mathrm{m}$-thick layer). It was assumed that the electric charges were localized in the space-charge region of the junction and that all the other regions of the structure were neutral. Furthermore, the diffusion component of the current in these regions was neglected. The $p-n$-junction has a nonlinear conductivity as well as nonlinear current-voltage dependence $J_{z}$ $=J_{s}[\exp (\mathrm{eV} / \beta k T)-1]$. Here, $J_{z}$ is the normal current density component, $J_{s}$ is the saturation current density, $V$ is the voltage drop in the junction, $\beta$ is the diode ideality factor, and $k$ is Boltzmann's constant. In this model, the potential and current distributions [the latter depends on the potential distribution because $\left.\vec{J}=-\sigma\left(r, z, J_{z}\right) \vec{\nabla} \varphi\right]$ are described by the equation $\vec{\nabla} \cdot\left[\sigma\left(r, z, J_{z}\right) \vec{\nabla} \sigma\right]=0$, where $\sigma\left(r, z, J_{z}\right)$ are the conductivities of LED layers. The boundary conditions are as follows: at the surfaces coated with metal contacts, the potential values are known, and at the other surfaces the normal current density component is zero. Even if such a simplified model does not enable the calculation of potential distribu- 

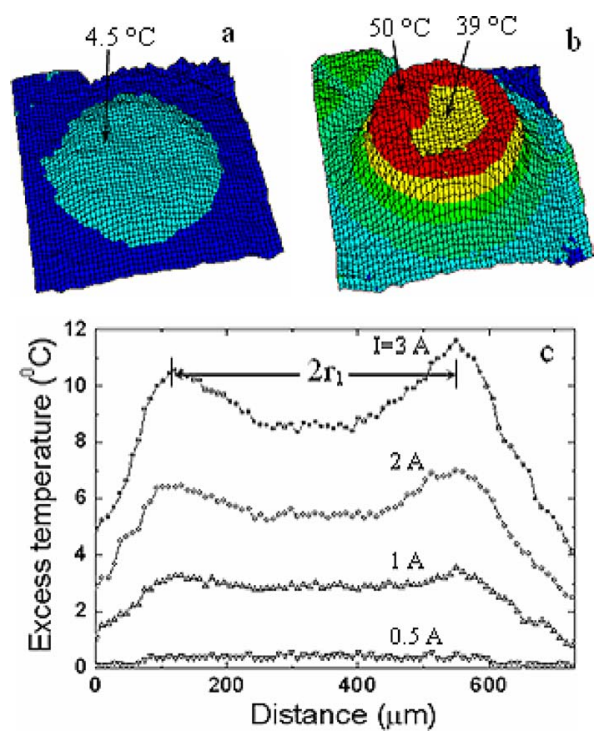

FIG. 2. (Color online) (a) Excess temperature pattern captured in cw (I $=350 \mathrm{~mA}$ ) mode and (b) under pulsed bias $I=3 \mathrm{~A}, \Delta t=160 \mathrm{~ms}$. (c) Excess temperature profile (along the diameter crossing, $10 \mu \mathrm{m}$ lateral step) at different currents, $\Delta t=400 \mu \mathrm{s}$. Neither the images nor the line plots have been processed or smoothed.

tion in the space-charge region, it correctly describes the distribution of the potential and current densities in other regions of the LED (in particular, in the active area, which captures most the interest).

The distribution of temperature $T(r, z, t)$ in the structure was determined by solving the nonstationary heat conduction equation. In this equation, the heat source was presented as $q=\left(J_{z}^{2}+J_{r}^{2}\right) /\left[\sigma\left(r, z, J_{z}\right)\right]$ (where $J_{r}$ is the radial current density component). The value of the power density of Joule losses was to be determined by solving the electric problem. The boundary conditions were as follows: at the outer boundaries (except at the bottom of the structure) the heat flows were zero, and at the bottom the heat flow was specified by the thermal resistance of the $p^{+}$-layer, which needed to be determined experimentally. For all the layers, the thermal conductivity was assumed to be $0.55 \mathrm{~W} / \mathrm{cm} \mathrm{K}$, regardless of the temperature. Furthermore, the possible temperature dependence of the conductivity was neglected.

\section{RESULTS AND DISCUSSION}

\section{A. LED active area self-heating}

The tests have shown that in cw mode, the heating of the active area starts at $I=350 \mathrm{~mA}\left(\Delta T=4.5^{\circ} \mathrm{C}\right)$ and increases considerably at higher currents. However, due to the diffusion of heat, the $\Delta T$ remains practically uniform over the entire active area [Fig. 2(a)].

The localization of heat source becomes possible only by using pulsed biasing. It turned out that at $I>1$ A and a pulse duration of $\Delta t=160 \mathrm{~ms}$, the active area heats up nonuniformly, and a "hot" ring forms in the active region opposite the central mesa contact. To illustrate this, at $I=3 \mathrm{~A}$, the temperature increases by $39{ }^{\circ} \mathrm{C}$ in the center and by $50{ }^{\circ} \mathrm{C}$ on the periphery of the active layer [Fig. 2(b)]. To determine the temperature gradient in the structure, local temperature measurements were taken with $10 \mu \mathrm{m}$ intervals. Figure 2(c)
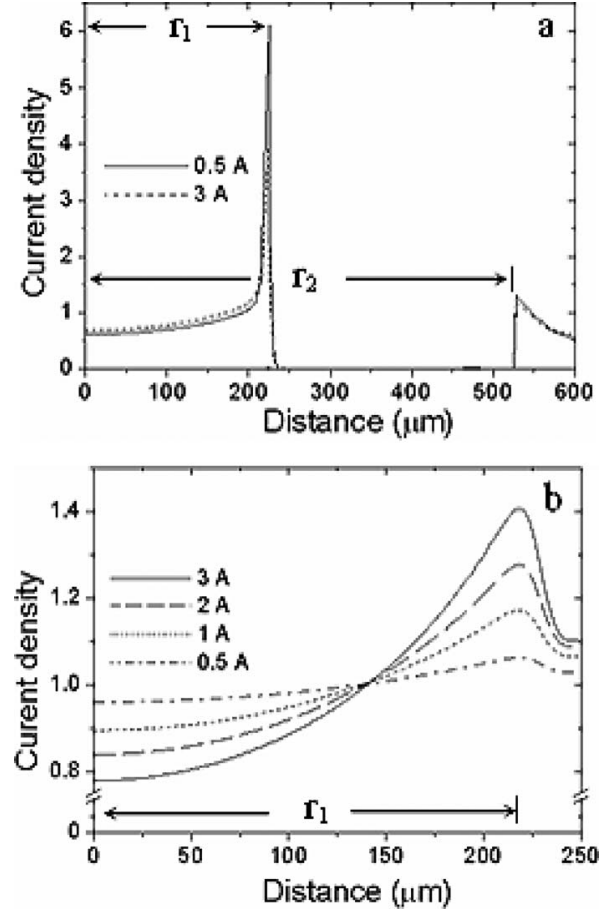

FIG. 3. (a) Normalized current density $\left(J_{z} / J\right)$ profiles (along the radius crossing) at the $p^{+}$-layer/bottom contact and $n$-layer/top contact interfaces and (b) in the active layer at different driving currents (theory).

shows the traces of 16 averaged heat profiles across the mesa. In this experiment, the current pulse duration $(\Delta t$ $=400 \mu s)$ was considerably lower than the characteristic time of thermal relaxation. It was found that temperature gradients on the periphery of the mesa reached 240,570 , and $950{ }^{\circ} \mathrm{C} / \mathrm{cm}$ at the driving currents of 1,2 , and $3 \mathrm{~A}$, respectively. For the purpose of comparison, the maximum temperature gradient at 160 -ms-long current pulse does not exceed $580^{\circ} \mathrm{C} / \mathrm{cm}$.

It was found that the high-peak pulsed current cycling leads to a random mechanical cracking at the peripheral region of the mesa, followed by the catastrophic degradation of the device. The heat map of this kind of a dead LED is shown in Fig. 5(b). Several local "Ohmic" conduction paths for current flow across the junction were detected through the JH of local areas, particularly at the location of the hot ring.

\section{B. Computer simulation of the CC features}

To identify the reason behind the nonuniform distribution of temperature in the LEDs, a computer simulation of the current flow was performed in different layers of the structure. The calculation results showed that the density of the current is nonuniform in the radial direction. Also, it was discovered that there are two types of $\mathrm{CC}$ located in different layers and differing in their dependence on the driving current.

Shown in Fig. 3 is the radial distribution of the current density $J_{z}$ at the bottom contact/ $/ p^{+}$-layer and top contact $/ n$-layer interfaces, normalized to the average current density under the corresponding contact (the contact CC effect). It is believed that this electrostatic effect is due to the singularity of the electric field at the abrupt metal- 

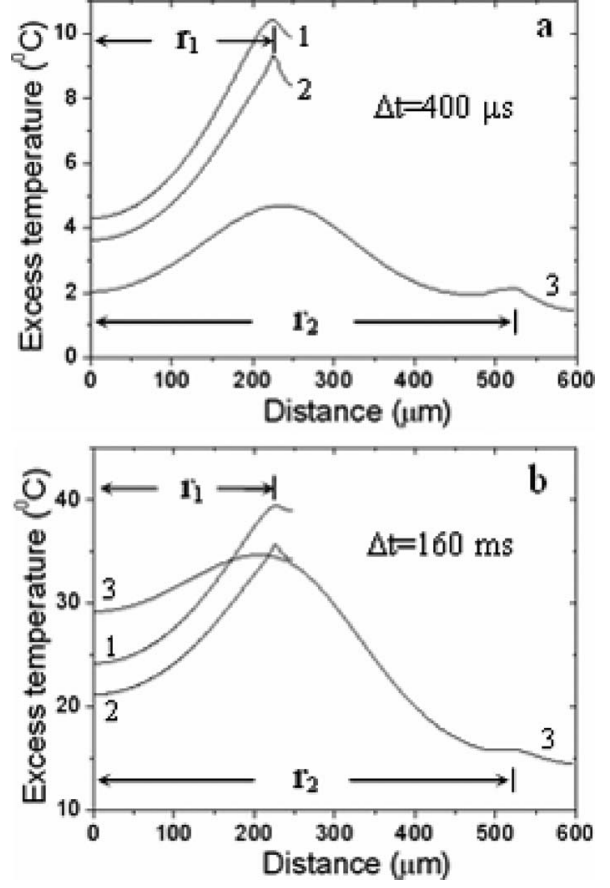

FIG. 4. Excess temperature profiles in different layers of a LED structure ( $I=3$ A, theory): (1) the active layer, (2) the bottom contact $/ p^{+}$-layer interface, and (3) the top surface.

semiconductor interface. ${ }^{15}$ Therefore, it depends on the driving current to some extent only. In the geometry we considered, this effect causes a sevenfold $(I>3$ A) increase in the current density at the bottom contact boundary, as compared to its value at the center. If the comparison is made to the average current density, then this value increases by a factor of 4.5 at the bottom contact boundary and decreases by $20 \%$ at the center. Calculations also show that the current crowds at the interface between the top contact and $n$-layer. However, due to the larger area of the top contact and the long distance between the contacts, the effect is less pronounced. Meanwhile, the current density along the inner perimeter of this contact is twice its value at the periphery.

The nonuniform distribution of the current density also forms in the active layer of the structure [Fig. 3(b)]. However, the $J_{z}$ in this region is distributed more evenly and remains practically uniform at $I<0.5$ A. At $I=3$ A, there is only a twofold increase in the $J_{z}$ at the periphery of the mesa, as compared to its value at the center, as well as an increase by $40 \%$ as compared to the average current density. Contrary to the contact $\mathrm{CC}$, an increase in the driving current in this region is accompanied by an essential redistribution of the current lines toward the periphery of the mesa that is a direct result of the nonlinear resistance of the junction (the $\mathrm{CC}$ at the active region). In fact, when crossing a junction, the current rout prefers the way of the lower resistance that is present on the peripheral part of the mesa. For example, the ratio between the peak current value and the value in the center does not exceed $110 \%$ at $I=0.5$ A, but it increases by almost two times its value at $I=3$ A.

Since the distribution of excess heat generated in the structure depends on the local current density, it is easy to reproduce the heat pattern using the finite element simulation. The results presented in Fig. 4 show the radial distribu-
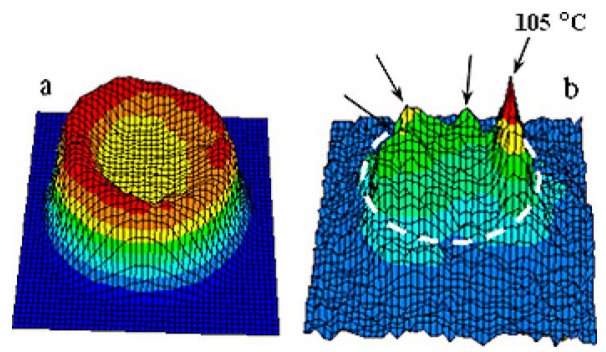

FIG. 5. (Color online) (a) Light pattern measured with CCD microscope at $I=3 \mathrm{~A}, \Delta t=160 \mathrm{~ms}$. (b) Heat map of a dead LED showing several shunting paths across the junction $(I=300 \mathrm{~mA}, \Delta t=160 \mathrm{~ms})$. The dotted circle is the projection of the bottom mesa contact.

tion of the JH in each layer of the LED structure at different durations of current pulses. The three anomalous temperature regions originating from two types of $\mathrm{CC}$ are easy to identify at the shorter pulse duration $\Delta t=400 \mu \mathrm{s}$ [Fig. 4(a)]. The first and most important heat source is the local heat concentration in the active layer (curve 1). It causes the light output to decrease due to the thermal and high-injection activation of nonradiative recombination of free charge carriers. Also, it is responsible for the thermal gradients inside the active layer of the structure (about $1200{ }^{\circ} \mathrm{C} / \mathrm{cm}$ at $I=3$ A) and for the nonuniform light pattern [Fig. 5(a)]. The second heat source with a slightly lower peak temperature forms in the $p^{+}$-cladding layer around the central mesa contact (curve 2). This is due to the contact CC effect and the series resistance of the $p^{+}$-layer. As the "emission depth" of radiation captured with the IR microscope is in the order of $p^{+}$-layer thickness, it is believed that the experimentally found temperature distribution shown in Fig. 2 is related to both of these effects. Finally, the third heat source is situated at the top of the LED surface $\left(r=r_{2}\right)$ and originates from the contact $\mathrm{CC}$ along the inner perimeter of the top contact (curve 3). The current density at this contact is low (because its surface is large), so the $\mathrm{CC}$ of this contact can be neglected. In fact, the smooth heat peak in the center of this curve $\left(r=r_{1}\right)$, which is a result of vertical heat diffusion from the active layer, causes stronger heating.

At the longer pulse duration ( $\Delta t=160 \mathrm{~ms})$, the shape of the lateral temperature distribution in each layer remains relatively unchanged. However, while the temperature scale increased, the thermal gradients decreased [Fig. 4(b)]. In fact, due to the process of heat diffusion, the temperature gradient in the active layer decreases from $1200^{\circ} \mathrm{C} / \mathrm{cm}$ $(\Delta t=400 \mu \mathrm{s})$ to $800{ }^{\circ} \mathrm{C} / \mathrm{cm}$ at $I=3 \mathrm{~A}$. For this reason and due to the nonsymmetric heat sink, the temperature in the center of the top layer exceeds the temperature in the active layer and the $p^{+}$-cladding layer.

A deeper look at the values of the thermal gradients in the active layer shows that the theoretical estimates always exceed the experimentally measured values. The reason for this discrepancy is the additional heat resulting from the nonradiative recombination of injected carriers that was neglected in our simulation. Indeed, due to the total internal reflection, only a small part of the radiation escapes the device. Most of this radiation degrades inside through multiple 
reabsorption, which is followed by nonradiative recombination. This randomly distributed heat decreases the experimentally measured thermal gradients.

\section{CC impact on the light output}

At $I<0.5$ A the light output detected with a CCD camera is evenly distributed in the active area. However, at $I$ $>1$ A, a bright "light crown" forms and its position coincides with the region of the $\mathrm{CC}$ in the active layer [Fig. 5(a)]. This type of situation, although it seems unusual at first (the light output should be lower in a more heated region), has a logical explanation. From the profile of the current density in Fig. 3(b), it can be concluded that at $I=3 \mathrm{~A}$, the CC leads to the current density redistribution, both on the periphery of the mesa and in the central region, in the ratio of 1.4/0.8. If the dependence of the light output on the bias current was linear, then the light power would change in the same ratio (the total power output is to remain the same). However, the experiment demonstrated only a 30\% increase in the light output at the periphery as compared to that in the center. In a word, the experiment indicates a decrease in the total power output that is caused by the CC and local heating (LED dynamic degradation).

Our tests showed that it is in the $\mathrm{CC}$ region of the active layer where the catastrophic degradation of the device happens. A durable operation of the device at a high current creates local shunts in the $p-n$-junction, which are easy to detect in IR. Furthermore, very hot spots were registered in the LEDs, which were dead shorts. As an example, Fig. 5(b) shows the heat map of a dead device biased with a moderate current. Enormous self-heating clearly shows that this degradation originates from the $\mathrm{CC}$ in the active region.

\section{CONCLUSION}

When driving currents well above the safe operating limit, the local $\mathrm{JH}$ and the nonuniform micropattern of light were detected in high-power large area AlGaAs LEDs. These effects originate from the two types of CC (in the active layer and the $p^{+}$-confining layer) and become especially critical in the extreme operating mode $(I>1 \mathrm{~A})$. It was found that they are easy to detect only by operating the LEDs in the pulsed mode on millisecond or shorter scales but they are masked by the heat diffusion in the longer pulsed or $\mathrm{cw}$ modes.

The CC in the active layer remarkably decreases the power conversion efficiency (reversible dynamic degradation due to the thermal quenching), reduces the in-plane emitting area, and forms a very nonuniform light beam. Most importantly, the CC develops the temperature gradient of about $950{ }^{\circ} \mathrm{C} / \mathrm{cm}$ at the periphery of the active layer. Since a heated volume element of the LED in the center expands less than a volume element further out, mechanical stresses in the radial direction appear. The stresses become most critical when the device is cycled between "on" and "off" states in the time scale that is shorter than the heat dissipation time. They may be responsible for the random catastrophic degradation of the device whose origin has not yet been determined.
Contact CC develops at an abrupt metal contact $/ p^{+}$-layer interface and causes the $p^{+}$-layer temperature to increase during the operation of the device. It also blocks the heat dissipation rout from the active layer toward the heat sink by increasing the risk of the degradation of the device. It is also important to note that this contact CC can cause the LED chip heat sink solder to soften or melt.

When designing high-power and large-area structures for dimming the LEDs operated in a pulse-width modulation mode,${ }^{16}$ these effects need to be fully understood, characterized, and taken into account. In particular, the $p^{+}$-cladding layer could be nominally doped and the metal contact needs to cover the entire surface of the mesa. A heat sink with the ability to selectively cool the local hot region ${ }^{17}$ (that follows the contact CC pattern) could also be helpful.

Finally, it is the overdrive mode that helped discover the way to avoid the limitations caused by the drop in efficiency in commercial high-power AlGaAs LEDs grown by liquidphase epitaxy. We have shown that the careful design of CC-free devices could considerably help increase the quantum efficiency of superhigh-power LEDs operated either in cw mode (when the carrier density in the active layer must be kept spatially uniform so that Auger recombination is minimized; IR illuminators; IR remote controllers) or in the pulse-width modulation mode (when the temperature gradients and mechanical stresses are a concern; high-speed IR communication).

\section{ACKNOWLEDGMENTS}

The work was funded by the Ministry of Education and Science within the Ukrainian-Russian Nanotechnology Project (Grant No. M/190-2007).

${ }^{1}$ I. Schnitzer, E. Yablonovitch, C. Caneau, and T. J. Gmitter, Appl. Phys. Lett. 62, 131 (1993).

${ }^{2}$ E. Dupont, C. H. Liu, M. Buchanan, S. Chiu, and M. Gao, Appl. Phys. Lett. 76, 4 (2000).

${ }^{3}$ P. Chambers, E. Austin, J. Gunning, and J. Dakin, Meas. Sci. Technol. 14, 2006 (2003).

${ }^{4}$ W. Fulop, Solid-State Electron. 14, 77 (1971).

${ }^{5}$ I. Schnitzer, E. Yablonovitch, C. Caneau, T. J. Gmitter, and A. Scherer, Appl. Phys. Lett. 63, 2174 (1993).

${ }^{6}$ X. Guo and E. F. Schubert, J. Appl. Phys. 90, 4191 (2001).

${ }^{7}$ V. Adivarahan, S. Wu, W. H. Sun, V. Mandavilli, M. S. Shatalov, G. Simin, J. W. Yang, H. P. Maruska, and M. Asif Khan, Appl. Phys. Lett. 85, 1838 (2004).

${ }^{8}$ R. M. Perks, A. Porch, and D. V. Morgan, J. Appl. Phys. 100, 083109 (2006).

${ }^{9}$ B. A. Matveev, M. A'daraliev, N. V. Zotova, S. A. Karandashev, M. A. Remenniy, N. M. Stus', G.N. Talalakin, V. K. Malyutenko, and O. Yu. Malyutenko, Proc. SPIE 4285, 109 (2001).

${ }^{10}$ V. Malyutenko, O. Malyutenko, A. Podoltsev, I. Kucheryavaya, B. Matveev, M. Remennyi, and N. Stus, Appl. Phys. Lett. 79, 4228 (2001).

${ }^{11}$ A. Monakhov, A. Krier, and V. V. Sherstnev, Semicond. Sci. Technol. 19, 480 (2004).

${ }^{12}$ V. Malyutenko, O. Malyutenko, A. Dazzi, N. Gross, and J.-M. Ortega, J. Appl. Phys. 93, 9398 (2003)

${ }^{13}$ V. K. Malyutenko, O. Yu. Malyutenko, and A. V. Zinovchuk, Appl. Phys. Lett. 89, 201114 (2006).

${ }^{14}$ V. Malyutenko, O. Malyutenko, A. Zinovchuk, A. Zakheim, D. Zakheim, I. Smirnova, and S. Gurevich, Proc. SPIE 5941, 308 (2005).

${ }^{15}$ B. Laikhtman, A. Gourevitch, D. Donetsky, D. Westerfeld, and G. Belenky, J. Appl. Phys. 95, 3880 (2004).

${ }^{16}$ P. Manninen and P. Orrevetelainen, Appl. Phys. Lett. 91, 181121 (2007).

${ }^{17}$ P. Wang and A. Bar-Cohen, J. Appl. Phys. 102, 034503 (2007). 